\title{
Un método para analizar la crisis en los sistemas educativos
}

A Method to Analyze the Crisis in the Educational Systems

Artículo de reflexión | Reflection Article

Fecha de recepción: 17 de julio de 2017 Fecha de aceptación: 18 de marzo de 2019 Fecha de disponibilidad en línea: marzo de 2020

doi: 10.11144/Javeriana.m13.macs

Antonio José Velasco antoniovelcas@gmail.com

Vector Consultoría, Venezuela (D) ORCID: https://orcid.org/0000-0001-8411-0263

Para citar este artículo | To cite this article Velasco, A. J. (2020). Un método para analizar la crisis en los sistemas educativos. magis, Revista Internacional de Investigación en Educación, 13, 1-26. doi: 10.11144/Javeriana.m13.macs 


\title{
Resumen
}

Por lo general, los estudios acerca de los sistemas educativos están basados en cifras sobre rendimiento, inversión, etc.; esto ha provocado que el concepto de crisis educativa haya permanecido poco claro conceptual y metodológicamente. En este artículo, se propone un método para su análisis, aplicable a distintos sistemas y niveles. Se expone una pauta de examen de los tipos de evaluación de la crisis educativa: evaluaciones directas, indirectas y mixtas; a continuación, se desarrolla un método para analizar uno de los tipos, basado en la investigación de las representaciones sociales. Finalmente, se presenta el modelo mediante una pauta metodológica y un instrumento.

\section{Palabras clave}

Investigación; metodología; sistema educativo; sistemas sociales

\begin{abstract}
Studies on the educational systems are generally based on figures of performance, investment, and so on. This has brought about that the concept of educational crisis still remains unclear both conceptually and methodologically. This article proposes a method to analysis this issue, applicable to different systems and levels. This work provides an examination standard for the types of evaluation of the educational crisis: Direct, indirect, and mixed evaluations. A method to analyze one of those types is developed herein, based on the research about the social representations. Finally, the model is set out with a methodological guideline and one instrument.
\end{abstract}

\section{Keywords}

Research; methodology; educational systems; social systems 
Descripción del artículo | Article description

En este artículo de reflexión, derivado del proyecto de investigación Métodos de investigación en ciencias humanas: epistemología y metodología, se presenta una propuesta teórica original acerca de los tipos de crisis de los sistemas educativos, la manera como se pueden analizar, y un método para llevar a cabo una de esas opciones, mediante el análisis de documentos.

\section{Introducción}

Un sistema educativo (SE) presenta diversas cuestiones importantes: número de alfabetizados, de técnicos, de graduados universitarios, de personas que han mejorado su vida gracias a la capacitación y formación de personal especializado, etc. Pero también, en muchos casos, se evidencian problemas y vicios, en especial en el mundo en desarrollo: problemas de corrupción, profesores que no asisten a su trabajo, nóminas de personal inexistente, profesores suplentes a quienes no se les paga por su trabajo, bajo rendimiento de egresados, inadecuación entre el diseño curricular y las necesidades del mercado laboral... (Bloque Social, 2006; Samoff, 1999; Subirats \& Nogales, 1989; Wangwe \& Rweyemamu, 2001). Y la principal preocupación de todo Estado, la reconozca o no: la excelencia de los egresados.

En el ámbito educativo, resulta recurrente hablar de crisis educativa (en adelante, $k$ ), pero, aun cuando ello se presente como diagnóstico, se suele dejar de lado la explicitación de los criterios empleados para evaluar el sistema educativo y aseverar que esté en crisis; en muchos casos, se habla de $k$, se la da a entender, o bien se la sugiere, sin analizar su fundamento; es usual la remisión a una (¿real o aparente?) crisis, con la cual todo se explica, o nada se logra explicar.

El espectro de posturas abarca desde quienes expresan la existencia de k (Anmol, 2015; Aparicio, 2011; Arcia \& Saltos, 1995; Coombs, 1978; Gómez, 1979; Lopes, 2015; Prats, 2005; Pretorius, 2015), hasta quienes la niegan (Martínez-García, 2006) para el caso de España; una postura diferente es la mantenida por Florentino Felgueroso, María Gutiérrez-Domènech y Sergi Jiménez-Martín (2014), y hay quienes, incluso analizando un sistema educativo, no la mencionan (Gasperini, 2000; diversos reportes de organismos internacionales), y aquellos que la suponen, mas no la consideran un problema relevante, o bien tratan solamente un aspecto crítico del sistema pues no es su tema central (Bobyleva \& Sidorova, 2015; Carr, 2012; Cedrés, s.f.; Dougherty, 2013; Kafer, 2007; Pastore, Sattar \& Tiongson, 2013; Raffe, Howieson \& Tinklin, 2002). 
El hecho de que en reportes internacionales $k$ no se maneje explícitamente como problema, o se considere solo alguna de sus aristas [en sus Informes de seguimiento de la educación para todos en el mundo (UNESCO, 2014), se trata el problema de la crisis del aprendizaje, no de la educación], hace pensar en problemas con su conceptualización, o que su polisemia genera problemas metodológicos.

Eso es así, porque se suele asumir la existencia de una crisis mas no se operacionaliza ese concepto; de modo que usualmente ni se lo analiza, ni se propone una manera de distinguir cuándo es que el SE entra en una verdadera crisis (total o parcial, temporal o constante) y cuándo funciona normalmente; de esta manera, la evaluación del sistema no solo suele quedar implícita sino además en un vacío conceptual y metodológico, lo cual convierte el concepto $k$ en poco útil, o bien puramente retórico.

A continuación, el objetivo es presentar una opción metodológica para el tratamiento de este problema y proponer un método para el análisis de $k$, sin presuponer sus causas ni sus consecuencias, pues el análisis de las representaciones sociales, RS, en el discurso no lo permite, y para ello se requiere de datos comportamentales e institucionales. El objeto de esta investigación no es la evaluación del SE, sino de la crisis del mismo. Se parte de una posición teórica abierta, favorable al enfoque metodológico mixto y al enfoque sistémico. El estudio que sigue es de naturaleza teórica, con intención aplicada, y su necesidad surge porque actualmente no hay un método como el que se propone.

El modelo propuesto se compone de dos partes: una primera parte teórica, en que se establecen las posibilidades de evaluación de un SE; y una segunda parte metodológica, que propone el método en cuestión.

Debido al espacio disponible, resulta imposible efectuar una rigurosa revisión conceptual de ciertos términos fundamentales, pero se pueden consultar las siguientes fuentes: Jaime Andréu-Abela (2000); Sandra ArayaUmaña (2002); Diccionario de la Real Academia Española de la Lengua (2018); Denise Jodelet (1988); Saadi Lahlou y Jean-Claude Abric (2011); Gordon Sammut, Eleni Andreouli, George Gaskell y Jaan Valsiner (2015); Tesauro UNESCO (2018).

\section{El sistema educativo}

Un SE se define como un tipo de sistema de naturaleza social, diseñado para la formación de los integrantes de la sociedad, que combina elementos materiales, comportamentales y simbólicos, y cuyo significado varía según el contexto cultural.

A pesar de las diferencias de insumos, de funcionamiento, de estructura e ideológicas en los SE internacionales, se los puede agrupar como 
tales debido a que cumplen funciones básicas de transmisión de información, formación de personal, transmisión de la cultura y modelización de la ciudadanía. Se debe partir de que todo SE es un sistema; se asume un enfoque general de sistemas aplicado a los educativos, como en los estudios de Frank Betts (1992); Regina Frei (2011); Theodore Frick, Kenneth R. Thompson y Joyce Koh (2008); Swati Gupta y Amit Gupta (2013); Sanaz Karim (2009); Selby Markham (s.f.) o Marco Aurelio Navarro Leal (1987).

\section{Evaluación de un sistema educativo}

\section{Elementos generales de evaluación}

La evaluación del SE puede asumir diversas formas: desde lo profundamente intuitivo hasta lo más concreto y cuantitativo, pasando por el análisis de redes (Álvarez-López, 2017; Jornet, López-González \& Tourón, 2012; Lizasoain-Hernández \& Joaristi-Olariaga, 2000; Miranda-López, 2013). Debido a limitaciones de espacio y para delimitar el tema, no se abordan aquí los lineamientos para la evaluación de los SE (aspectos a evaluar, indicadores, contextualización, etc.).

En el caso de muchos SE, su evaluación suele resultar muy complicada debido a que cada SE se ve afectado severamente por la influencia del sistema político (SP), del que forma parte, de manera que los procesos políticos del país presentan correlatos en el SE, lo que significa que es un modelo que reproduce los problemas, confrontaciones y vicios del SP — sirva como ejemplo el caso de Venezuela (Gouveia, 1985) —, existiendo también argumentos en contra, y a favor de la relativa autonomía de los SE en América Latina (Fernando Reimers en Cordero, 1999).

En todo caso, una suposición acerca de la existencia de aspectos críticos dentro del SE supone la evaluación del mismo, y ello requiere considerar dos elementos: uno, la evaluación de ese algo con respecto a un estado, situación o proceso normal; y, en correspondencia, el otro es la existencia de un canon, regla, norma o pauta, que establece qué es correcto y qué no, y sobre la base de esa consideración, se podrá decir que algo está o no en un estado crítico.

En cualquier caso, resulta necesario contar con un canon de evaluación o valoración de los parámetros del sistema, y con un rango de valores entre los cuales se acepta que el sistema funcione normalmente, lo cual requiere entonces considerar ciertas unidades de medida.

Así, se podría decir que si un SE determinado produce (genera, ocasiona, etc.) en uno de sus niveles 300 unidades de $Y$ (graduados, por ejemplo) durante un tiempo $\Delta t$ con un insumo de 20 unidades de $X$ (docentes), $y$ la norma establece que debería generar un mínimo de 550 y un máximo de 
650 unidades para ese lapso, con ese insumo y bajo las condiciones $a, b$ y $c$, entonces el sistema no está funcionando correctamente, y posiblemente esté en crisis.

Se notará que los términos de uso común en este terreno, como "aceptable", "importante", "ordinario"..., remiten a un concepto de fondo, el de normalidad, que es la clave para evaluar la dinámica del sistema. Y ese concepto incluye al menos dos sentidos: (a) el de lo que es aceptado por quienes manejan el sistema, por sus beneficiarios, por quienes forman parte del mismo, o por quienes lo evalúan, y (b) el de lo que es aceptable debido a que se establece formal u oficialmente como si fuera una norma (como las leyes, reglamentos, resoluciones, etc.).

\section{Opciones de evaluación}

Evaluar un SE puede ser considerado desde dos puntos de vista. Uno en el que se recurre a medidas determinadas objetivamente, que se denominarán evaluaciones directas; y otro en el cual se acude a estimaciones, esto es, a valoraciones subjetivas, que se denominarán aquí evaluaciones indirectas.

La primera clase de evaluaciones presenta dos opciones:

1. Implica que se desea comparar uno o más aspectos del SE con respecto a una pauta o canon (una normativa, por ejemplo), cánones, parámetros o estándares, usualmente indicadores de producto en cifras nacionales, como asistencia a la escuela primaria, capacidad lectora, escolarización o correlación entre escolaridad y niveles de aprendizaje: se le llamará Evaluación Tipo 1.

2. Implica que se evaluará el sistema con respecto a un modelo empírico (de la misma naturaleza que el evaluado, pero que quizás lo hace peor o mejor que este), o bien uno teórico. Esta se denominará Evaluación Tipo 2.

Se debe decir que la Evaluación tipo 1 implica la existencia de una norma (canon) o parámetros, y que la tipo 2 implica no solamente la existencia de una norma o normas, sino también de una similitud que permita la operación comparativa con el sistema real.

A esta primera clase de evaluaciones se les denominará Evaluaciones directas del sistema educativo, por cuanto en ellas se recurre a indicadores objetivos de funcionamiento del sistema, los cuales atañen directamente a sus componentes, estructura, procesos, insumos o productos. Así, en el caso de las Evaluaciones directas Tipo 1 de un SE, el SE se podrá evaluar con respecto a la norma que establece la Ley de Educación del país, la Constitución de ese país, o cualquier ley nacional que establezca parámetros de 
medida (real o supuesta) de lo que es deseable para el SE, o asimismo, cualquier estándar internacional de medición, como la eficacia o la eficiencia de un sistema educativo; este es el caso de pruebas estandarizadas como PISA (Programme for International Student Assessment, o Programa Internacional para la Evaluación de Estudiantes, en matemáticas, lectura y ciencias).

Y en el caso de las Evaluaciones directas Tipo 2, esto es, por comparación con un modelo, el SE se podrá evaluar comparándolo con otro análogo, como el español, esloveno, francés, cubano, estadounidense, etc. O bien, con un modelo no empírico sino teórico, como el Índice de Desarrollo de la Educación para Todos (IDE) de la UNESCO (índice compuesto de evaluación global del SE de un país en relación con los objetivos de Educación Para Todos - EPT_- un movimiento mundial guiado por la UNESCO para satisfacer necesidades de aprendizaje de todos los niños, jóvenes y adultos para 2015).

No obstante, hay tres problemas típicos con esta primera clase de evaluaciones, aunque pretenden ser objetivas: uno es que la norma suele ser sumamente abstracta cuando es de rango legal, y ello tiene como consecuencia que quizás no haya claridad en cuanto a la metodología a ser aplicada, y las evaluaciones necesariamente se harán sobre una base extremadamente abstracta o global.

Otro inconveniente es que cuando la pauta no es tan abstracta, entonces suele estar restringida a aspectos sumamente concretos del sistema (es el caso de las resoluciones y circulares ministeriales), lo cual tiene un efecto contrario al anterior, y las evaluaciones efectuadas sobre esa base pueden carecer de la generalidad necesaria.

Y el tercer problema es que cuando se acude a pruebas estándar internacionales, por lo general se evalúan solamente productos o resultados, como la cantidad de egresados por nivel, el desempeño de los egresados en ciertos aspectos (razonamiento verbal o matemático, por ejemplo). Además, dado que las condiciones de funcionamiento de cada SE nacional y local influyen sobre el aprendizaje del sujeto, y que los insumos afectan los procesos y los resultados obtenidos por los egresados de uno u otro sistema, esto hace que comparaciones aparentemente lícitas en realidad no necesariamente lo sean. De allí la inconveniencia de efectuar comparaciones que el ente investigador no aprueba ni promueve, si los insumos y condiciones de funcionamiento de los SE son diferentes, ya que los desempeños de los estudiantes deberán serlo también, y resultan no comparables, salvo que se lleven a cabo correcciones en los datos que permitan controlar la influencia de los factores referidos; aunque ello, no siempre es posible.

La segunda clase de evaluaciones son denominadas Evaluaciones indirectas del SE, puesto que en ellas no se acude a indicadores directos y objetivos de funcionamiento real del sistema, sino a valoraciones subjetivas del 
mismo; esto es, a la apreciación de personas que guardan alguna relación con el SE. Y estas apreciaciones subjetivas a su vez tienen (también) una relación necesaria con algún tipo de canon o norma, puesto que se formulan en términos de deseabilidad: Ios sujetos que valoran o critican el SE lo hacen teniendo como punto de referencia alguna idea, concepto o patrón (una norma o canon) acerca de lo que es bueno para la sociedad y de cómo debería funcionar, en consecuencia, el SE.

La diferencia fundamental entre las dos clases de cánones mencionados, esto es, los empleados para las Evaluaciones objetivas (Tipo 1 y 2), y los utilizados para las valoraciones subjetivas (los demás tipos, que se verán ahora) es que en la primera clase se trata de normas establecidas objetivamente o reconocidas como tales (a saber, una pauta en la ley, un estándar internacional o el rendimiento de un sistema similar), mientras que en el segundo grupo se trata de estándares de deseabilidad, individual o social.

Debido a ello, la primera clase de cánones ya ha sido establecida "objetivamente" por así decir, mientras que los cánones de la segunda clase han de ser establecidos mediante el análisis de las preferencias de la población o grupos de referencia; por ello, en el presente trabajo se sugerirá luego el análisis de las representaciones sociales.

En todo caso, las valoraciones subjetivas del SE atañen a todas las estimaciones subjetivas puntuales de su funcionamiento, tales como las opiniones acerca de su eficiencia por parte de expertos, las actitudes hacia el sistema por parte de la población, las valoraciones de calidad del egresado en distintos niveles por parte del mercado laboral, etc. Así, esta segunda clase de evaluaciones presenta varios tipos, a saber:

1. Por una parte, se puede llevar a cabo el análisis de opiniones de legos en la materia acerca del SE, y estas opiniones pueden ser de individuos, de grupos o del colectivo nacional: es la Evaluación Tipo 3 (lo que suele hallarse en los reportajes periodísticos y encuestas de opinión).

2. Por otra parte, puede implicar que el sistema se evalúa mediante la valoración por estimaciones subjetivas que del SE formulen individuos o grupos, los cuales usualmente serán expertos en la materia, o Evaluación Tipo 4 (es lo que suele encontrarse en las entrevistas a formadores de opinión sobre la materia).

3. Y en tercer término, están los análisis de los modelos y concepciones, esto es, las representaciones que acerca del sistema, de su funcionamiento, o de aspectos específicos del mismo (como $k$ ) tiene un sector dado de la población, a lo que usualmente se le denomina como representaciones sociales, la Evaluación Tipo 5. 
Por otro lado, es importante tener en cuenta que también se dan casos en que el sujeto evaluador utiliza indistintamente varias clases de cánones de evaluación; a esto se le denominará evaluaciones mixtas del sistema, o Evaluación Tipo 6. Esto ocurre cuando en el juicio acerca del SE, a pesar de ser (obviamente) subjetivo u opinable, se recurre o se pretende estar recurriendo a un canon objetivo. En este caso, lo típico es que un experto recurra a medidas objetivas de rendimiento y funcionamiento para brindar soporte a sus ideas previas, juicios que suelen estar apuntalados por el uso — parcial o no, técnicamente correcto o no- de estadísticas —oficiales o no- acerca del rendimiento del SE.

En la tabla 1 se resumen los tipos indicados, con ejemplos de la literatura especializada.

Tabla 1

Tipos de evaluación del sistema educativo: ejemplos

\begin{tabular}{|c|c|c|c|}
\hline \multirow[t]{2}{*}{$\begin{array}{l}\text { Evaluaciones } \\
\text { directas }\end{array}$} & \multirow[t]{2}{*}{$\begin{array}{l}\text { Con indicadores } \\
\text { objetivos de } \\
\text { funcionamiento } \\
\text { del sistema }\end{array}$} & $\begin{array}{l}\text { Tipo } 1 \\
\text { Con relación a un } \\
\text { canon, cánones } \\
\text { o parámetros }\end{array}$ & $\begin{array}{l}\text { Gustavo Arcia y Ángel Saltos, } 1995 . \\
\text { Bloque Social, } 2006 . \\
\text { Alla Bobyleva y Alexandra Sidorova, } 2015 . \\
\text { Florentino Felgueroso, María Gutiérrez-Domènech } \\
\text { y Sergi Jiménez-Martín, } 2014 . \\
\text { Krista Kafer, } 2007 . \\
\text { José Saturnino Martínez-García, } 2006 . \\
\text { Joaquim Prats, } 2005 . \\
\text { David Raffe, Cathy Howieson y Teresa Tinklin, } 2002 . \\
\text { Joel Samoff, } 1999 .\end{array}$ \\
\hline & & $\begin{array}{l}\text { Tipo } 2 \\
\text { Con relación a un } \\
\text { modelo empírico } \\
\text { o teórico }\end{array}$ & $\begin{array}{l}\text { Vivek Kumar Anmol, } 2015 . \\
\text { Philip H. Coombs, } 1978 . \\
\text { Marco Aurelio Navarro-Leal, } 1987 . \\
\text { Jacques van der Gaag y Pauline Abetti, } 2011 . \\
\text { Informes de seguimiento de la educación en } \\
\text { el mundo de la UNESCO. }\end{array}$ \\
\hline \multirow[b]{2}{*}{$\begin{array}{l}\text { Evaluaciones } \\
\text { indirectas }\end{array}$} & \multirow[b]{2}{*}{$\begin{array}{l}\text { Valoraciones } \\
\text { subjetivas del } \\
\text { sistema }\end{array}$} & $\begin{array}{l}\text { Tipo } 3 \\
\text { Opiniones de legos } \\
\text { acerca del sistema }\end{array}$ & $\begin{array}{l}\text { Reportes de prensa, sondeos de opinión } \\
\text { pública y similares. }\end{array}$ \\
\hline & & $\begin{array}{l}\text { Tipo } 4 \\
\text { Estimaciones } \\
\text { subjetivas del } \\
\text { sistema por } \\
\text { expertos }\end{array}$ & $\begin{array}{l}\text { Pablo Christian Aparicio, } 2011 . \\
\text { Edward R. Dougherty, } 2013 . \\
\text { Silvia Finocchio, } 2010 . \\
\text { Víctor Manuel Gómez, } 1979 . \\
\text { José Gouveia, } 1985 . \\
\text { José David Lara-González, } 2012 . \\
\text { Maria Lopes, } 2015 .\end{array}$ \\
\hline \multirow{2}{*}{$\begin{array}{l}\text { Evaluaciones } \\
\text { mixtas }\end{array}$} & \multirow{2}{*}{$\begin{array}{l}\text { Varias clases } \\
\text { de cánones de } \\
\text { evaluación }\end{array}$} & $\begin{array}{l}\text { Tipo } 5 \\
\text { Análisis de las } \\
\text { representaciones } \\
\text { del sistema }\end{array}$ & $\begin{array}{l}\text { Stephanus Gert Pretorius, } 2015 . \\
\text { Eduardo Santa Cruz-Grau y Antonio Olmedo, } 2012 . \\
\text { María del Carmen Cedrés, s.f. }\end{array}$ \\
\hline & & Tipo 6 & $\begin{array}{l}\text { Nicholas Carr, } 2012 . \\
\text { José Subirats e Ivonne Nogales, } 1989 . \\
\text { Samwel M. Wangwe y Dennis C. Rweyemamu, } 2001 .\end{array}$ \\
\hline
\end{tabular}




\section{Un método para el análisis de la crisis del SE}

El estudio de la crisis de un sistema social altamente complejo como un SE puede hacerse siguiendo dos grandes rutas: la evaluación directa de si existe o no una $k$, lo que produce una estimación bastante objetiva de lo que se llamará Crisis real del SE ( $k$ real), mediante evaluaciones de la primera clase. Y la otra ruta es la evaluación indirecta, basada en las percepciones de funcionamiento del mismo, de acuerdo con indicadores acerca de estados subjetivos de valoración del sistema, los cuales sería de esperar que se encontrasen estandarizados, pero esto es mucho menos probable, y este análisis produce como resultado una estimación más o menos objetiva de lo que aquí se denominará Crisis percibida del SE (k percibida).

La diferencia entre ambas formas de $k$ está clara: una cosa es que realmente haya alguna clase de crisis en el sistema, y otra diferente es que se crea en ello. Se presentan cuatro posibilidades: (a) existencia de crisis y percepción de la misma, (b) no existencia de crisis, pero percepción de que la hay, (c) no existencia de crisis y ausencia de creencia en ella, y (d) existencia de crisis real, pero sin percepción de esta (tabla 2).

Tabla 2

Percepción versus existencia de crisis en el sistema educativo

\begin{tabular}{l|l|l}
\hline Percepción de crisis & Existencia de crisis & \\
\hline & Existe & No existe \\
\hline Es percibida & Crisis real percibida & Percepción de crisis inexistente \\
\hline No es percibida & Crisis real no percibida & No existe crisis ni se la percibe \\
\hline
\end{tabular}

Fuente: elaboración propia

Por lo demás, hay que tener presente que los juicios acerca de la existencia de la crisis real en un SE deberían estar claramente circunscritos por el analista a aquel aspecto y lapso en el cual se presenta la supuesta situación crítica. La razón para ello es que hablar de crisis general del SE tiene muy poca utilidad y que, de haber una verdadera crisis generalizada y prolongada del SE, este difícilmente pudiera haber existido más allá de cierto límite temporal, con lo que el sistema probablemente ya habría colapsado.

\section{El concepto de crisis educativa}

Como se ha indicado previamente, en el concepto de "crisis", la existencia de un canon de valoración resulta indispensable para su análisis en alguno de los aspectos de un sistema cualquiera, debido a que afirmar la 
existencia de una crisis (parcial o total, leve o severa, temporal o permanente), en realidad implica que ya se sabe cuáles son (o se conocen los efectos de) los estados no críticos del sistema.

Aquí se definirá la crisis como un fenómeno de disrupción severa y persistente en el funcionamiento o en un estado de un sistema. Por disrupción, se entiende toda situación del sistema que incluya una interrupción de la actividad normal en alguno de sus componentes. Por severidad de la disrupción, se entiende que la misma afecta de un modo importante el sistema; el grado de importancia dependerá del parámetro que se establezca (sea estándar o convencional); por ejemplo, que afecte el funcionamiento en un $80 \%$, en un $50 \%$... Por persistencia de la disrupción, se entiende que se mantiene durante un lapso mayor al que sería de esperar, o mayor al que resulta aceptable, lo que será establecido, bien convencionalmente, bien objetivamente. Por funcionamiento, se entiende el cumplimiento de los procesos ordinarios del sistema, para los fines que le hayan sido establecidos, durante un lapso dado. Por estado, se entiende cualquier situación de los componentes del sistema, para un momento dado.

Ahora bien, hay que tener presente que una crisis no es una ruptura, quiebre o destrucción del sistema, sino un estado previo a una posible ruptura que podría no ocurrir; además, no es algo negativo per se, puesto que puede ser una reacción temporal del sistema para afrontar un problema, un ataque o una emergencia. Así, parece tener tres alternativas de continuidad: el paso a un deterioro no reversible; la recuperación del área que estaba en crisis, y la desaparición de dicha área; en los tres casos la crisis, obviamente, finaliza.

De acuerdo con lo expuesto, la crisis educativa $(k)$ consiste en la disrupción severa y persistente en el funcionamiento o en un estado de un sistema educativo.

\section{El análisis de la crisis educativa percibida mediante Evaluaciones de tipo 5}

Ahora se formula una propuesta metodológica para el análisis de la $k$ percibida del SE, para Evaluaciones de tipo 5, sobre la base del discurso de un sector de la población. El estudio de María del Carmen Cedrés (s.f.) fue de encuesta y entrevistas a maestros acerca de representaciones sobre sus alumnos, evaluación y fracaso escolar, y representaciones como ex alumnos. El trabajo de Felicita Garnique C. (2012) fue un estudio cualitativo de docentes, con observación participante, revisión de documentos, cuestionario y entrevistas. Luz María Martínez de Correa (2001) efectuó un estudio comparativo del discurso institucional por modalización del discurso y campos 
semánticos, analizando elementos políticos y económicos. Stephanus Gert Pretorius (2015) hizo una investigación cualitativa inductiva acerca de las percepciones de docentes sobre la naturaleza de la crisis y su contexto, para generar un modelo sobre el liderazgo y gerencia de la crisis educativa. María del Mar Rodríguez-Romero (2000) analizó cualitativamente las metáforas del cambio educativo y su vinculación con las representaciones del mismo. Mercedes Valentina Salazar-Gómez y María Teresa Herrera-Aponte (2008) hicieron un estudio hermenéutico de la estructura de las RS de docentes de Educación básica. El trabajo de Eduardo Santa Cruz-Grau y Antonio Olmedo (2012) realizó un análisis cualitativo de discurso periodístico.

\section{El rol de los elementos discursivos}

El análisis de —o la determinación de si existe- una percepción de $k$ requiere partir de la presencia de objetos referenciales en el discurso de los actores (A) seleccionados, objetos como el Estado, los docentes, la familia, la Iglesia, los opositores, los partidos, el Gobierno, etc., y dado que cada A evalúa de diferente modo la influencia de cada uno de tales objetos sobre el fenómeno $k$, es necesario analizar el papel que juega cada uno de esos objetos de referencia en el discurso del actor con respecto al concepto $k$.

A cada objeto referencial en el discurso de un A se le denominará elemento (e), y se debería analizar el papel que el A le asigna en su discurso, en dos sentidos: uno, su influencia en cuanto a la generación (o reducción) de la crisis, o Causalidad del e: un e puede así ser un factor causal de $k$, puede ser parte de un proceso causal en $k$, o bien puede ser un efecto de $k$. $Y$ otro, su papel en cuanto a la severidad de su influencia sobre la crisis, o Criticidad del e, esto es: un e puede ser considerado como generador de $k$, como un elemento neutro en cuanto a $k$, o bien como un atenuador o paliador de $k$.

Una vez esto sea determinado, se puede proceder al examen de la Relevancia que en el texto producido por A presenta cada elemento en cuanto a $k$, esto es, la relación que existe entre su presencia en el texto, su criticidad y su relevancia causal, lo cual por lo general no aparecerá explícitamente determinado en el discurso del A, sino que será obtenido por el analista al examinar la relación entre los aspectos indicados, como se explicará más adelante.

Además de examinarse la relación que guardan los elementos discursivos como principales puntos de referencia en el discurso de cada $A$, debe analizarse también el modo como cada A trata el concepto $k$, y considerar dos cuestiones: una, la frecuencia del concepto $k(f k)$ en el discurso del A, y otra, la intensidad emotiva asociada a este concepto $(A d)$.

La fk surge de un conteo de las referencias directas al concepto en cada documento, y la Ad se estima mediante el examen de la adjetivación 
asignada por A al concepto en cada aparición del mismo, mediante alguna adjetivación: esto es lo referente a su valoración de la crisis como algo más o menos bueno o malo (juicio de valor) y como algo más o menos severo; al efecto, se puede emplear un diferencial semántico para establecer la intensidad emotiva de los adjetivos.

El discurso de los sujetos puede ser recolectado de diversas fuentes: entrevistas, textos escritos por los sujetos, documentos oficiales del grupo o institución, comunicados, etc. El análisis deberá arrojar la identificación de dos cuestiones cruciales: una, cuáles son los aspectos o temas alrededor de los cuales se construye el discurso de la crisis, los Ejes temáticos; y otra, las agrupaciones de elementos que presentan modelos subjetivos de carácter causal acerca de la crisis según el discurso de los actores, los Nodos críticos.

\section{Las representaciones sociales}

Una representación social (RS) es un producto simbólico complejo, comúnmente no intencional, de un sector o de toda una sociedad, y resultado de un proceso de construcción social usualmente no dirigido (ArayaUmaña, 2002; Jodelet, 1988; Moñivas-Lázaro, 1994). En tal sentido, la investigación de RS amerita su reconstrucción por el analista, y esta, dado que normalmente es elaborada sobre la base de discursos de actores sociales, requiere de la identificación de los elementos que configuran la representación (discusiones en Lahlou \& Abric, 2011; Parales-Quenza \& Vizcaíno-Gutiérrez, 2007). Una postura las divide en Núcleo, Esquema figurativo y Sistema periférico, y acá se la sigue, sin desmedro de otras alternativas de estructuración de las RS (Sammut, Andreouli, Gaskell \& Valsiner, 2015).

Uno de los tipos de análisis más interesantes para Evaluaciones de tipo 3 y tipo 5 (percepción y representación de $k$ ) es el de las RS referidas al SE (Garnique, 2012; Martínez de Correa, 2001; Rodríguez-Romero, 2000; Salazar-Gómez \& Herrera-Aponte, 2008): las RS pueden mostrar el estado que presenta la imagen del sistema o cómo ha evolucionado esta para una población dada: maestros, directores, padres, estudiantes, etc.

\section{Herramientas de trabajo: algoritmo de análisis}

Seguidamente, se propone un procedimiento heurístico o algoritmo para el análisis de la $k$ percibida, en el discurso de actores sociales pertenecientes a un SE cualquiera. Varios de los pasos (en especial 13, 14 y 15) pueden ser efectuados según la técnica o técnicas de análisis de contenido que prefiera el analista (Alonso-Benito \& Callejo-Gallego, 1999; Andréu-Abela, 2000; Piñuel-Raigada, 2002), siempre que sea utilizada consistentemente — si es la misma - y coherentemente — si son varias-. 
I. Acopio: 1. Redactar una lista de criterios de selección de actores sociales relacionados con el tema de interés (como ideología, pertenencia a grupos determinados, edad, sexo, etc.). 2. Seleccionar de la lista a los actores sociales más relevantes para el tema. 3. Hacer acopio de la documentación contentiva del discurso de los actores, mediante el muestreo adecuado, o mediante la selección de toda la producción discursiva pública de los actores; debe tratarse de documentos públicos y de amplia difusión social, pues en otro caso se trataría de una representación no social, sino privada y restringida. 4. Crear criterios de inclusión de los documentos: provenientes de una fuente de circulación nacional (no restringida a círculos exclusivos, como periódicos o documentos internos de organizaciones), no anónimos, con discurso atribuible claramente a un A, o a distintos actores dentro de un mismo documento en diferentes discursos, y con alusión por parte del $\mathrm{A}$ al tema de $k$ en al menos una ocasión en cada documento o cada discurso. 5. Catalogar los documentos según sus emisores (los A) y los fenómenos considerados críticos.

II. Análisis: 6. Realizar un primer análisis de documentación: elaborar una lista de posibles objetos referenciales que se pueden encontrar en el discurso, sobre la base de los criterios de selección considerados, siendo los primeros elementos componentes primarios de la RS del SE. 7. Desarrollar un segundo análisis de documentos: identificar los principales elementos (e) del discurso. 8. Llevar a cabo un tercer análisis de documentación: identificar los principales objetos referenciales en el discurso de los actores. 9. Determinar la criticidad para cada e, según su papel en los fenómenos críticos, según cada $A$, según cada agrupación de $A$, y en general. 10. Establecer la causalidad para cada e según su papel en los fenómenos críticos, en cuanto al rol causal que juega cada e en la generación de la crisis: según cada A, según cada agrupación de $A$, y en general. 11. Realizar un análisis de la relevancia para cada e.

III. Representación de $k$ : 12. Establecer la estructura de los elementos de RS: relevancia e importancia. 13. Identificar los ejes temáticos en el discurso para cada documento y para cada A. 14. Identificar los nodos críticos en el discurso para cada documento y para cada A. 15. Reconstruir el concepto de $k$ según los datos obtenidos. 16. Reconstruir la RS de k: Núcleo, Esquema figurativo y Sistema periférico.

\section{Operacionalización de las variables}

\section{Ideología política del actor}

Afiliación ideológica de cada A, emisor de discurso. Pudieran clasificarse como oficialistas, opositores y neutrales o no identificados con ninguno de ambos bloques. 
Relevancia del elemento discursivo ( $\mathrm{R} e$ )

Relación entre la causalidad, la criticidad y la frecuencia de cada e de la RS en el discurso de los A. Se calcula mediante la suma algebraica de los valores en causalidad $(R c)$ y criticidad $(C e)$, multiplicada por el valor de la frecuencia (fe). Fórmula: $R e=(R c+C e)^{*} f e$.

\section{Relevancia del concepto k. (Rk)}

Relación entre la adjetivación y la frecuencia de aparición de $k$ en el discurso de los actores. Se refiere a los adjetivos calificativos asociados por el actor a $k$. Se calcula mediante la sumatoria de los valores en adjetivación del concepto $\left(\sum_{1}^{n} A d\right)$, dividida por el valor de la frecuencia de aparición del concepto de crisis (fk). Fórmula: $R k=\sum_{1}^{n} A d / f k$.

\section{Eje temático (ET)}

Tema que surge de los distintos discursos de los $A$ y destaca por que alrededor de él se configura narrativamente la mayor carga representacional: ejemplos de ello pueden ser la democracia, la dictadura, la corrupción, el caudillismo, el Estado, el partidismo, etc.

\section{Nodo crítico (NC)}

Agrupaciones de dos o más e que muestran una estructura causal. Se trata de un complejo discursivo referido a un tema ( $k$, en este caso) compuesto por dos o más e interrelacionados, hacia el cual convergen dos o más factores causales y desde el cual parten dos o más efectos.

\section{Importancia del elemento en la representación social (IeRS)}

Valor subjetivo para cada elemento en la representación social de $k$. Se calcula mediante la multiplicación de la frecuencia del elemento $(f e)$ en la muestra de discursos por el número de actores que comparten el elemento (NAe). Fórmula: leRS $=f e^{\star} N A e$.

\section{Pertenencia del elemento a la representación social}

Porcentaje del total de los $A$ que comparten el elemento. Se calcula mediante una tabla porcentual, para la que se propone la siguiente escala: de $0-29 \%=$ No pertenece a la RS; entre $30-79 \%$ = Pertenece a la Periferia de la RS y de $80-100 \%=$ Pertenece al Núcleo de la RS.

Los elementos de análisis propuestos son los mínimos necesarios para un discernimiento adecuado de k; empero, cada analista podrá disminuir la cantidad de aspectos considerados. Ciertamente, hay otras variables de interés que pueden ser estudiadas, aunque las que se han referido parecen las fundamentales. Esas otras variables pudieran ser atinentes a la 
pertenencia a un partido político, al cargo que ocupa el funcionario oficialista, a la organización a que pertenece el opositor, entre otras, incluso la edad y sexo de los actores.

\section{Un instrumento para el análisis de la crisis educativa en el discurso}

Finalmente, se presenta el instrumento propuesto para llevar a cabo el análisis. Se muestra en las siguientes figuras, cada una correspondiente a una página del instrumento. Para la determinación de su validez, se sugiere el juicio de expertos (análisis racional de ítems) y validez convergentediscriminante (si se dispone de instrumentos similares) y para determinar confiabilidad, las pruebas piloto.

Figura 1

Portada del instrumento: descripción, instrucciones y Parte I

\begin{tabular}{|c|}
\hline Planilla para el análisis de documentos \\
\hline [Título de la investigación] \\
[Responsable de la investigación] \\
[Institución]
\end{tabular}

\section{A. Descripción}

Propósito del instrumento: Captar los elementos básicos (e) de la representación de la crisis educativa en documentos contentivos del discurso de formadores de opinión o actores $(\boldsymbol{A})$, e identificar el modo como se maneja el concepto de crisis educativa $(\boldsymbol{k})$ en cada documento analizado.

\section{B. Instrucciones para el/la analista}

- Los aspectos que aparecen marcados con numerales en las casillas de cada tabla del presente instrumento son las variables a ser utilizadas en la creación de una basede datos para el vaciado de estos.

- $\quad$ Antes de proceder a efectuar el análisis, se debe leer el Glosario del presente instrumento.

- Una vez efectuado el análisis, deberá llenarse lo siguiente:

\begin{tabular}{|l|l|l|l|l|}
\hline $\begin{array}{l}\text { Código del/la } \\
\text { analista }\end{array}$ & Fecha & & & Firma \\
\hline
\end{tabular}

Parte I. Datos básicos del documento

\begin{tabular}{|l|l|l|l|l|}
\hline 1. Código & $\begin{array}{l}\text { Título del } \\
\text { documento }\end{array}$ & $\begin{array}{l}\text { 3. Actor } \\
\text { 4. Ideología } \\
\text { del actor }\end{array}$ & Derecha Neutra & Izquierda \\
\hline 2. Tema & & & \\
\hline
\end{tabular}

\begin{tabular}{|l|l|l|l|}
\hline Fecha & & Fuente \\
\hline
\end{tabular}


Figura 2

Parte // del instrumento, página 2

Parte II. Identificación de los elementos de la representación de la crisis educativa en el documento

1. Copiar en la primera columna de la izquierda las cláusulas o fragmentos pertinentes del documento y luego resaltar los elementos (e) de la representación social de la crisis y el concepto de crisis educativa $(\boldsymbol{k})$ en cada fragmento del discurso analizado.

2. El elemento puede destacarse mediante resaltado en amarillo y el concepto de crisis educativa y sus adjetivos mediante resaltado en verde.

3. Subrayar en la primera columna los adjetivos que sean adjudicados al concepto de crisis en cada fragmento analizado.

4. Anotar en la segunda columna cada elemento encontrado en cada fragmento.

5. En la tercera columna, indicar cuál es el rol del elemento hallado en cuanto a su causalidad sobre la crisis educativa, esto es, el Rol causal del e en cuanto a la crisis educativa, como Factor causal, Proceso causal o Efecto (ver Glosario).

6. En la cuarta columna, indicar cuál es el carácter que en el discurso del Actor analizado asume cada e en cuanto a la crisis educativa, esto es, la Criticidad del e (ver Glosario).

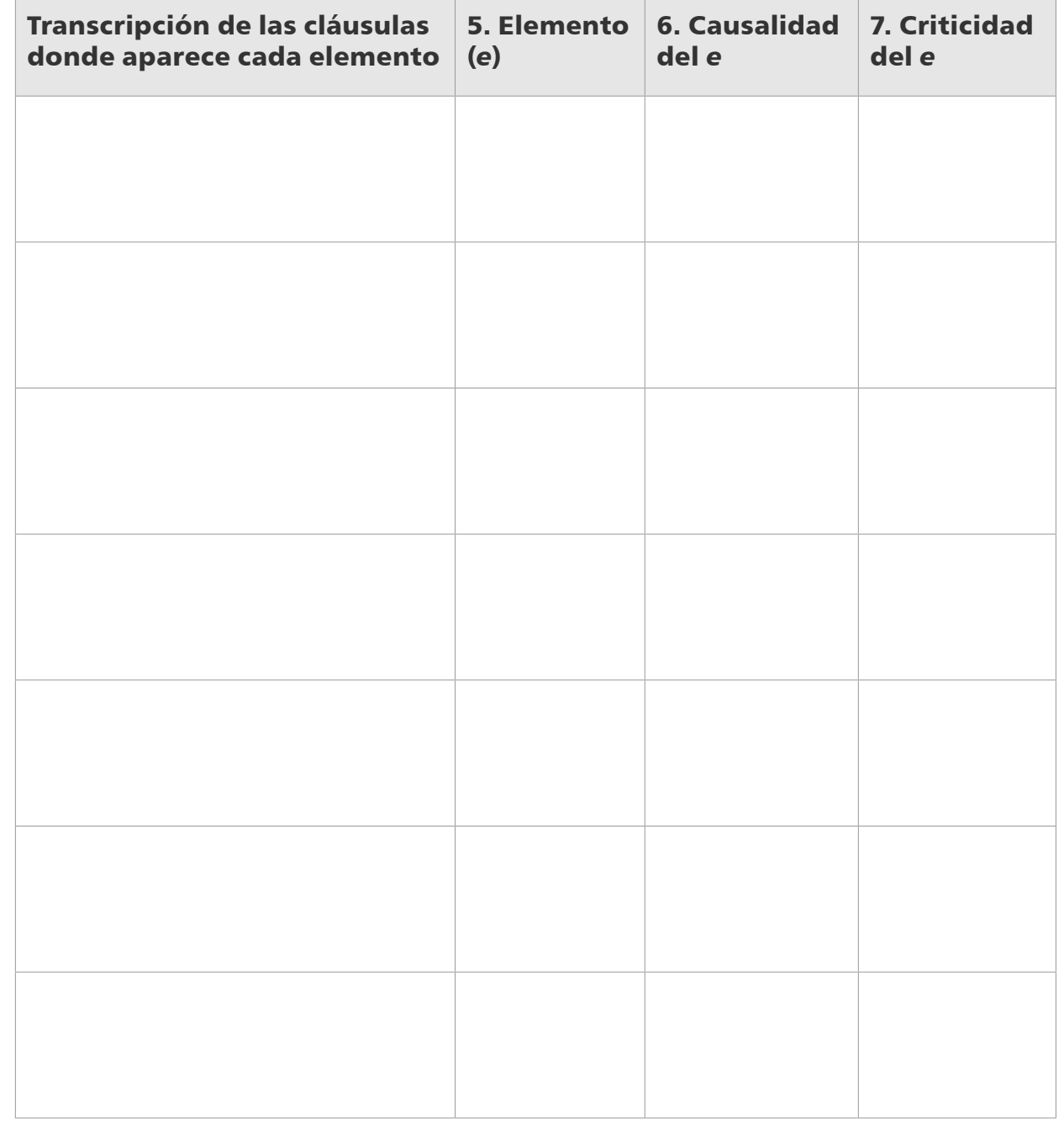

Fuente: elaboración propia 
Figura 3

Parte III del instrumento, página 3

Parte III. Cálculo de la relevancia de cada elemento presente en el documento

1. Anotar en la segunda columna de la izquierda cada uno de los elementos hallados en el documento analizado.

2. En la tercera columna, anotar la frecuencia de aparición de cada elemento en el documento $(\boldsymbol{f e})$.

3. En la cuarta columna, asignar el puntaje en Causalidad a cada elemento identificado $(\boldsymbol{R C})$, indicando el puntaje correspondiente al rol causal de cada elemento, mediante lasiguiente clave: Factor causal (FC): 3 puntos. Proceso causal (PC): 1 punto. Efecto (EF): 2 puntos

4. En la quinta columna, asignar puntaje a cada elemento en cuanto a su Criticidad $(\mathbf{C e})$, Ilenando cada columna según la clave: Generador de crisis ( $k+)$ : 1 punto. Neutro en cuanto a la crisis (k0): 0 puntos. Paliador de crisis (k-): -1 punto.

5. En la sexta columna, calcular la relevancia de cada elemento en cuanto a la crisis educativa $(\boldsymbol{R e})$, según la fórmula $\boldsymbol{R e}=(\boldsymbol{R c}+\mathbf{C e}) * \mathbf{f e}$

\begin{tabular}{|c|c|c|c|c|c|}
\hline & Elemento & $\begin{array}{l}\text { 8. Frecuencia } \\
\text { del e (fe) }\end{array}$ & $\begin{array}{l}\text { 9. Causalidad } \\
\text { del e (Rc) }\end{array}$ & $\begin{array}{l}\text { 10. Criticidad } \\
\text { del e (Ce) }\end{array}$ & $\begin{array}{l}\text { 11. Relevancia del e } \\
\operatorname{Re}=(\boldsymbol{R c}+\mathrm{Ce})^{*} \mathrm{fe}\end{array}$ \\
\hline 1 & & & & & \\
\hline 2 & & & & & \\
\hline 3 & & & & & \\
\hline 4 & & & & & \\
\hline 5 & & & & & \\
\hline 6 & & & & & \\
\hline 7 & & & & & \\
\hline 8 & & & & & \\
\hline 9 & & & & & \\
\hline 10 & & & & & \\
\hline 11 & & & & & \\
\hline 12 & & & & & \\
\hline 13 & & & & & \\
\hline 14 & & & & & \\
\hline Etc. & & & & & \\
\hline
\end{tabular}

Fuente: elaboración propia 
Figura 4

Parte IV del instrumento, página 4

Parte IV. Cálculo de la relevancia de la crisis educativa en el documento

Acá ya no se analiza el tratamiento de los elementos en el discurso,

sino cómo se adjetiva la crisis educativa en el discurso del actor analizado

1. Anotar en la columna izquierda cada uno de los adjetivos que aparecen asociados al concepto de crisis educativa $(\boldsymbol{k})$ en el documento analizado (ver Diferencial semántico al final).

2. Asignar un puntaje a cada uno de esos adjetivos según la escala (0 a 4 puntos: de Ninguna a Gravísima), haciendo una marca en la columna que corresponda.

3. Totalizar en la última fila de la tabla el puntaje de adjetivación del concepto de $\operatorname{crisis}(\boldsymbol{A d})$.

4. Efectuar la sumatoria de los puntajes de cada columna en la casilla para la sumatoria de la adjetivación de $\boldsymbol{k}$.

5. Anotar la frecuencia con que el concepto de crisis educativa aparece en el documento analizado, en la casilla para Frecuencia de $\boldsymbol{k}$.

6. Calcular la relevancia del concepto $\boldsymbol{k}$ en el documento $(\boldsymbol{R} \boldsymbol{k})$ según la siguiente fórmula: $R k=\sum_{1}^{n} A d / f k$. Esto significa: dividir el total de la sumatoria entre la frecuencia de aparición del concepto de crisis educativa.

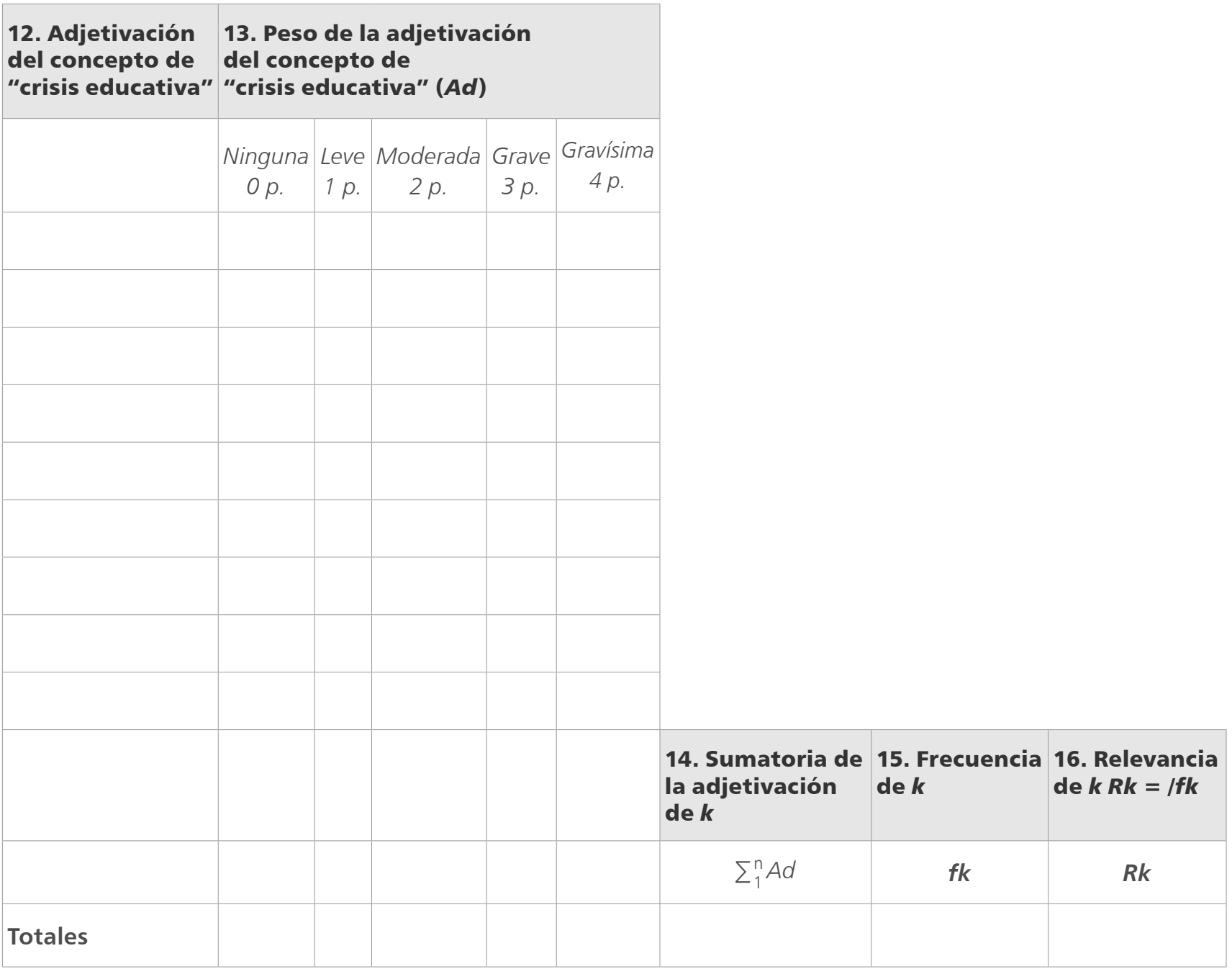

Fuente: elaboración propia 
Figura 5

Glosario para el uso del instrumento, página 5

\section{Glosario}

1. Actor: sujeto autor del contenido del documento analizado, o emisor del discurso allí contenido.

2. Adjetivación del concepto de "crisis educativa": modo como el actor califica la crisis, según los adjetivos que utilice.

3. Crisis educativa $(\boldsymbol{k})$ : disrupción severa y persistente en el funcionamiento, o en un estado, de un sistema educativo.

4. Criticidad del e: papel que juega cada elemento en la crisis; puede ser un generador de crisis $\left(\mathbf{k}^{+}\right)$, un e neutro en cuanto a la crisis $\left(\mathbf{k}^{0}\right)$ debido a que no causa ni elimina la crisis, o bien un paliador de la crisis $\left(\mathbf{k}^{-}\right)$: pues no causa la crisis, sino que la evita o la atenúa.

5. Efecto (EF): no es un e causal de la crisis, sino uno que es producto de la crisis.

6. Elemento de la representación social (e): cualquier cosa que según el discurso analizado resulte relevante a efectos de la crisis educativa o del funcionamiento del sistema educativo analizado. Puede tratarse de una persona, de un país, de un sistema, de un fenómeno natural, de una agrupación, etc.

7. Factor causal (FC): es un e que interviene directamente en la crisis educativa.

8. Frecuencia del e en el documento $(\boldsymbol{f e})$ : número de ocasiones en que aparece cada e en el documento analizado.

9. Frecuencia de $\boldsymbol{k}$ : cantidad de veces que el actor menciona el concepto o la idea de crisis educativa.

10. Peso de la adjetivación del concepto de "crisis educativa" $(\boldsymbol{A d})$ : valor del adjetivo asignado por el actor al concepto $\boldsymbol{k}$ cada vez que se refiera al mismo.

11. Proceso causal (PC): es un e no único sino plural (2 o más e) que se manifiesta a lo largo del tiempo.

12. Relevancia de cada e (Re): puntaje obtenido por cada e en el análisis del documento.

13. Rol causal o causalidad del e: esto se refiere al papel que juega cada e identificado en cuanto a la generación o presencia de $\boldsymbol{k}$ : puede ser un e que interviene directamente en la crisis educativa (factor causal), puede tratarse de un e no único sino complejo que se manifiesta a lo largo del tiempo (proceso causal) o bien no ser un e causal de la crisis, sino que es un producto de la crisis (efecto).

14. Sumatoria de la adjetivación de $\boldsymbol{k}$ : suma del total del peso de la adjetivación del concepto de crisis.

15. Relevancia de $\boldsymbol{k}$ : resultado numérico de dividir el total de la sumatoria de la adjetivación de la crisis en el documento por la frecuencia de aparición del concepto de crisis en dichodocumento. 
Figura 6

Términos para el diferencial semántico, página 6 Términos para el diferencial semántico de la adjetivación
del concepto de "crisis educativa"

\begin{tabular}{|l|l|l|l|l|}
\hline Ninguna $\mathbf{p}$ p. & Leve $\mathbf{2}$ p. & Moderada $\mathbf{p}$. & Grave 4 p. & Gravísima 5 p. \\
\hline No & Suave & Media & Marcada & Terrible \\
\hline No existe & Ligera & Mediana & Enorme & Tremenda \\
\hline No hay & Breve & Parcial & Severa & Extraordinaria \\
\hline Nunca & A veces & Eventual & Intensa & Inaudita \\
\hline Jamás & Ocasionalmente & Superable & Dura & Increíble \\
\hline Inexistente & Quizás & Soportable & Muy seria & Extrema \\
\hline Falsa & Simple & Llevadera & Alta & Bárbara \\
\hline Ninguna & Fácil & Necesaria & Pésima & Permanente \\
\hline Ausente & Alguna & Parcial & Fuerte & Insuperable \\
\hline Exento de & Pasajera & Normal & Mucha & Total \\
\hline Imposible & Difusa & Localizada & Completa & Inimaginable \\
\hline Inconcebible & No preocupante & Aceptable & Extensa & Absoluta \\
\hline Carente de & Leve & & Magnificada & Definitiva \\
\hline
\end{tabular}

Fuente: elaboración propia

\section{Conclusión}

En este documento se ha presentado una propuesta metodológica para un análisis de la crisis educativa. Tal análisis - mientras no se base en la evidencia, esto es, en un estudio desapasionado y no partidista, con evaluaciones de tipo 1 y 2 - no irá más allá de las evaluaciones de tipo 3 y 6 o, a lo sumo, de tipo 4. Esto deja en evidencia que los dos primeros tipos deben ser acometidos con frecuencia - por parte de tesistas, por ejemplo-y que sus resultados deberían recibir mayor difusión entre el público, quizás sobre todo entre el personal del ministerio del ramo.

La proposición de operacionalizar y medir variables e indicadores no pretende que se deba dejar de lado lo cualitativo, en absoluto, puesto que para evaluar cualquier sistema social el análisis cualitativo es imprescindible. Tampoco se propone aquí matematizar un sistema tan complejo como el $\mathrm{SE}$, solo se matematiza una nueva forma de análisis del asunto. Este modelo metodológico deberá ser criticado, aplicado sobre textos concretos, corregido y modificado por los expertos, para verificar su utilidad para la investigación. 


\section{Sobre el autor}

Antonio José Velasco es doctor en Educación. Profesor invitado en universidades de Venezuela y UNED, España. Miembro del consejo editorial de The Qualitative Report, Revista Latinoamericana de Análisis Existencial, Revista Electrónica de Psicología Política. Árbitro de Magis, Journal of Artificial Societies and Social Simulation, The Qualitative Report, Revista Electrónica de Psicología Política, Interamerican Journal of Psychology.

\section{Referencias}

Alonso-Benito, L. E. \& Callejo-Gallego, J. (1999). El análisis del discurso: del postmodernismo a las razones prácticas. Reis: Revista Española de Investigaciones Sociológicas, 88, 37-73. Disponible en: https://dialnet.unirioja.es/servlet/ articulo?codigo $=759360$

Álvarez-López, G. (2017). Descentralización educativa y evaluación de sistemas educativos: conceptualización y análisis del caso español. RIESED: Revista Internacional de Estudios sobre Sistemas Educativos, 2 (7), 52-73. Disponible en: http://www.riesed.org/index.php/RIESED/article/view/102/121

Andréu-Abela, J. (2000). Las técnicas de análisis de contenido: una revisión actualizada. Centro de Estudios Andaluces, 10 (2), 1-34. Disponible en: http://mastor.cl/blog/wp-content/uploads/2018/02/Andreu.-analisis-decontenido.-34-pags-pdf.pdf

Anmol, V. K. (2015). Value Based Education and Today Crisis. International Journal of Multidisciplinary Research and Development, 2 (1), 47-49. Disponible en: http://www.allsubjectjournal.com/archives/2015/vol2/issue1

Aparicio, P. (2011). Crisis de la reforma educativa argentina en un contexto neoliberal. Los handicaps de la inclusión educativa y laboral juvenil. Actualidades Investigativas en Educación, 11 (1), 1-29. Disponible en: https://revistas.ucr. ac.cr/index.php/aie/article/view/10171/18028

Araya-Umaña, S. (2002). Las representaciones sociales: ejes teóricos para su discusión. San José, Costa Rica: Facultad Latinoamericana de Ciencias Sociales, FLACSO, Cuadernos de Ciencias Sociales, 127. Disponible en: http://unpan1. un.org/intradoc/groups/public/documents/ICAP/UNPAN027076.pdf

Arcia, G. \& Saltos, Á. (1995). Ecuador - Análisis de la crisis educativa. Bases para un consenso. Quito: Reporte para Research Triangle Institute.

Betts, F. (1992). How Systems Thinking Applies to Education. Educational Leadership, 50 (3), 38-41. Disponible en: http://www.ascd.org/publications/edu cational-leadership/nov92/vol50/num03/How-Systems-Thinking-Appliesto-Education.aspx

Bloque Social (2006). La crisis educativa en Chile. Diagnóstico y propuestas. Observatorio de Educación. Santiago de Chile: Bloque Social. Disponible en: http://opech.cl/bibliografico/calidad_equidad/Documento_Bloque_So cial_Noviembre.pdf

Bobyleva, A. \& Sidorova, A. (2015). Crisis Management in Higher Education in Russia. Internationalisation in Higher Education: Management of Higher Education and Research, 3 (1), 23-35. PRADEC Interdisciplinary Conference 
Proceedings. Disponible en: http://academicpublishingplatforms.com/down loads/pdfs/picp/volume4/201508200159_03_PICP_Vol3_Issue1_2015_Bob yleva_and_Sidorova_Crisis_management_higher_education_Russia_pp.2335.pdf

Carr, N. (2012). The Crisis in Higher Education. MIT Technology Review, NovemberDecember. Disponible en: http://www.technologyreview.com/featuredstory/ 429376/the-crisis-in-higher-education/

Cedrés, M. (s.f.). Un estudio de las representaciones sociales de los maestros sobre el fracaso escolar. Tesis de maestría en Políticas y Administración de la Educación, Buenos Aires: Universidad Nacional Tres de Febrero. Disponible en: http://www.untref.edu.ar/documentos/tesisposgrados/Cedres.pdf

Coombs, P. H. (1978). La crisis mundial de la educación. Barcelona: Provenza.

Cordero-Arroyo, G. (1999). Educación, pobreza y desigualdad. Entrevista a Fernando Reimers. Revista Electrónica de Investigación Educativa, REDIE, 1 (1), 72-80 Disponible en: http://www.redalyc.org/pdf/155/15501106.pdf

Dougherty, E. R. (2013). On the Impoverishment of Scientific Education. Journal on Bioinformatics and Systems Biology, 15, 1-11. doi: 10.1186/1687-41532013-15

Felgueroso, F.; Gutiérrez-Domènech, M. \& Jiménez-Martín, S. (2014). Dropout Trends and Educational Reforms: the Role of the LOGSE in Spain. IZA Journal of Labor Policy, 3 (9), 1-24. DOI: 10.1186/2193-9004-3-9. Disponible en: https://link.springer.com/content/pdf/10.1186\%2F2193-9004-3-9.pdf

Finocchio, S. (2010). Una cartografía de saberes escolares en movimiento para América Latina. Propuesta Educativa, 19 (34), 2, 65-76. Disponible en: http:// www.propuestaeducativa.flacso.org.ar/archivos/dossier_articulos/46.pdf

Frei, R. (2011). A Complex Systems Approach to Education in Switzerland. En T. Lenaerts (ed.). Advances in Artificial Life, ECAL 2011: Proceedings of the Eleventh European Conference on the Synthesis and Simulation of Living Systems, 242-249. Cambridge, MIT. Disponible en: https://archive.org/de tails/ECAL2011

Frick, T. W.; Thompson, K. R. \& Koh, J. (2008). Predicting Education System Outcomes: A Scientific Approach. En M. Orey, V. McClendon \& R. Branch (eds.). Educational Media and Technology Yearbook, 33, 62-76. Disponible en: https://www.indiana.edu/ tedfrick/aect2006/PESO.pdf

Gaag, J. van der \& Abetti, P. (2011). Using National Education Accounts to Help Address the Global Learning Crisis. Washington, D.C.: Brookings Institution. Disponible en: https://www.brookings.edu/wp-content/ uploads/2016/06/0421_national_education_vandergaag.pdf

Garnique, F. (2012). Las representaciones sociales. Los docentes de la educación básica frente a la inclusión escolar. Perfiles Educativos, XXXIV (137), 99-118. Disponible en: http://www.scielo.org.mx/pdf/peredu/v34n137/v34n137a7.pdf Gasperini, L. (2000). The Cuban Education System: Lessons and Dilemmas. Washington, The World Bank. Country Studies, Education Reform and Management Publication Series, 1 (5). Disponible en: https://siteresour ces.worldbank.org/EDUCATION/Resources/278200-109907987726 9/547664-1099080026826/The_Cuban_education_system_lessonsEn 00.pdf 
Gómez, V. M. (1979). Crisis, interpretación y prospectiva del desarrollo educativo en América Latina. Revista Latinoamericana de Estudios Educativos, IX (4), 71-88. Disponible en: http://www.cee.edu.mx/rlee/revista/r1971_1980/r_ texto/t_1979_4_04.pdf

Gouveia, J. N. (1985). Construyamos un nuevo sistema educativo. Tertulia con Ernesto Mayz-Vallenilla. Nosotros, marzo, 8-11. Disponible en: http://www. dsm.usb.ve/mayz/_archivos_pdf/nosotros_tertuliaconemv_fotos.pdf

Gupta, S. \& Gupta, A. (2013). The Systems Approach in Education. International Journal of Management, 1 (1), 52-55. Disponible en: http://www.mitpubli cations.org/yellow_images/1388230198_logo_paper\%207.pdf

Jodelet, D. (1988). La representación social: fenómenos, conceptos y teoría. En S. Moscovici. Psicología social, vol. II, Capítulo 13, 469-493. Barcelona: Paidós.

Jornet, J.; López-González, E. \& Tourón, J. (2012). Evaluación de sistemas educativos: teoría y experiencia. Bordón, 64 (2), 9-11. Disponible en: https://dialnet. unirioja.es/descarga/articulo/3960088.pdf

Kafer, K. (2007). Taking the Boy Crisis in Education Seriously: How School Choice Can Boost Achievement among Boys and Girls. Position Paper No. 604, April. Independent Women's Forum. Disponible en: http://www.iwf.org/fil es/49ba4dcb1f95dacf6d20aa721e429c88.pdf

Karim, S. (2009). Applying Systems Approach to Educational - Organizational Change - Improvement of an Interdisciplinary Program. Master Program in Sustainable Development, Uppsala University. Disponible en: http://uu.divaportal.org/smash/get/diva2:393282/FULLTEXT01.pdf

Lahlou, S. \& Abric, J.-C. (2011). What Are the "Elements" of a Representation? Papers on Social Representations, 20, 20.1-20.10. Disponible en: http:// www.psych.Ise.ac.uk/psr/, http://eprints.Ise.ac.uk/44355/1/What\%20 are\%20the\%20\%E2\%80\%9Celements\%E2\%80\%9D\%20of\%20a\%20 representation(Isero).pdf

Lara-González, J. D. (2012). ¿Fracaso educativo, educación fracasada en México? Nómadas, Revista Crítica de Ciencias Sociales y Jurídicas, Número Especial: América Latina, 89-110. http://dx.doi.org/10.5209/rev_NOMA.2012.41770. Disponible en: http://dx.doi.org/10.5209/rev_NOMA.2012.41770

Lizasoain-Hernández, L. \& Joaristi-Olariaga, L. (2000). El análisis de datos en la evaluación de programas educativos. Revista de Investigación Educativa, 18 (2), 357-379. Disponible en: https://revistas.um.es/rie/article/down load/121041/113731/

Lopes, M. (2015). A crise da escola: o (re)pensar de uma outra escola face aos desafios do século XXI. Revista Ibero-americana de Educação, 69 (1), 183198. Disponible en: http://www.rieoei.org/deloslectores/6990.pdf

Markham, S. (s.f.). A SocioCybernetic Approach to Educational Systems. Colloquium: A Model for Integrating Instructional Technology into Higher Education. Disponible en: http://www.csse.monash.edu.au/ smarkham/reading/ SocioCybernetic.pdf

Martínez de Correa, L. M. (2001). Representaciones sociales de los discursos político-educativos de Venezuela y Cuba. Cuestiones Políticas, 26, 34-59. Disponible en: produccioncientificaluz.org/index.php/cuestiones/article/ download/14193/14172 
Martínez-García, J. S. (2006). La falsa crisis del sistema educativo. Témpora: Revista de Historia y Sociología de la Educación, 9 (diciembre), 85-103. Disponible en: https://josamaga.webs.ull.es/crisis-sistema-educativo.pdf

Miranda-López, F. (2013). La evaluación a debate. Entre la calidad y la desafiliación de los jóvenes de la educación secundaria en América Latina. Revista Latinoamericana de Educación Comparada, 4 (4), 41-58. Disponible en: https://dialnet.unirioja.es/descarga/articulo/6503072.pdf

Moñivas-Lázaro, A. (1994). Epistemología y representaciones sociales: concepto y teoría. Revista de Psicología General y Aplicada, 47 (4), 409-419. Disponible en: http://dialnet.unirioja.es/servlet/articulo?codigo $=2385297$

Navarro-Leal, M. A. (1987). El análisis de sistemas y la crisis permanente de la educación. Revista de la Educación Superior, 16, 2-62. Disponible en: http:// publicaciones.anuies.mx/pdfs/revista/Revista62_S1A1ES.pdf, http://publicaciones.anuies.mx/journal/62/1/1/es/el-analisis-de-sistemas-y-la-crisis-permanente-de-la-educacion

Organización de las Naciones Unidas para la Educación, la Ciencia y la Cultura, UNESCO (2014). Enseñanza y aprendizaje: Lograr la calidad para todos. 110. Informe de Seguimiento de la EPT [Educación para Todos] en el Mundo. París: UNESCO. Disponible en: http://unesdoc.unesco.org/images /0022/002261/226159s.pdf

Organización de las Naciones Unidas para la Educación, la Ciencia y la Cultura, UNESCO (2018). Tesauro. París: UNESCO. Disponible en: http://vocabularies. unesco.org/browser/thesaurus/es/

Parales-Quenza, C. J. \& Vizcaíno-Gutiérrez, M. (2007). Las relaciones entre actitudes y representaciones sociales. Revista Latinoamericana de Psicología, 39 (2), 351-361. Disponible en: http://www.redalyc.org/pdf/805/80539210. pdf, http://www.scielo.org.co/pdf/rlps/v39n2/v39n2a10.pdf

Pastore, F.; Sattar, S. \& Tiongson, E. (2013). Gender Differences in Earnings and Labor Supply in Early Career: Evidence from Kosovo's School-to-Work Transition Survey. IZA Journal of Labor \& Development, 2 (5), 1-34. DOI: 10.1186/2193-9020-2-5

Piñuel-Raigada, J. L. (2002). Epistemología, metodología y técnicas del análisis de contenido. Estudios de Sociolingüística, 3 (1), 1-42. Disponible en: https:// www.ucm.es/data/cont/docs/268-2013-07-29-Pinuel_Raigada_AnalisisCon tenido_2002_EstudiosSociolinguisticaUVigo.pdf

Prats, J. (2005). VII. El sistema educativo español. En J. Prats \& F. Raventós (dirs.). Los sistemas educativos europeos. ¿Crisis o transformación?, 177-228. BarceIona: Fundación La Caixa. Disponible en: http://www.ub.edu/histodidactica/ images/documentos/pdf/sistemas_educativos_europeos_crisis_transforma cion.pdf

Pretorius, S. G. (2015). Education in Crisis: First Steps towards a Crisis Management Model for South Africa's Dysfunctional Schools. $3^{\text {rd }}$ International Conference on Innovation, Documentation and Teaching Technologies, INNODOCT Conference, 13-15 May, Spain, 88-96. Valencia: Universitat Politècnica de València. Disponible en: https://riunet.upv.es/bitstream/ handle/10251/70950/PDF-Dema\%3bGarrig\%C3\%B3s\%3bPeris\%20 -\%20INNODOCT.\%203rd\%20INTERNATIONAL\%20CONFERENCE\%20 
ON\%2OINNOVATION\%2C\%20DOCUMENTATION\%20AND\%20TEA.... pdf? sequence $=1 \&$ is Allowed $=y$

Raffe, D.; Howieson, C. \& Tinklin, T. (2002). The Scottish Educational Crisis of 2000: An Analysis of the Policy Process of Unification. Journal of Education Policy, 17 (2), 167-185. Disponible en: http://www.research.ed.ac.uk/portal/en/pu blications/the-scottish-educational-crisis-of-2000-an-analysis-of-the-policyprocess-of-unification(db4aece8-c7cd-4762-b9a4-f6c0bbbe8be9).html

Rodríguez-Romero, M. del M. (2000). Las representaciones del cambio educativo. Revista Electrónica de Investigación Educativa, REDIE, 2 (2), 23-46. Disponible en: https://redie.uabc.mx/redie/article/view/23/45

Salazar-Gómez, M. V. \& Herrera-Aponte, M. T. (2008). Representaciones sociales de los valores educativos y prácticas pedagógicas. Laurus, Revista de Educación, 14 (26), 347-366. Disponible en: http://www.redalyc.org/ pdf/761/76111491017.pdf

Sammut, G.; Andreouli, E.; Gaskell, G. \& Valsiner, J. (2015). Cambridge Handbook of Social Representations. Cambridge: Cambridge University Press.

Samoff, J. (1999). No Teacher Guide, No Textbooks, No Chairs: Contending with Crisis in African Education. 43 $3^{\text {rd }}$ Annual Meeting of the African Studies Association, Philadelphia, 1-14 November. Disponible en: https://eric. ed.gov/?id=ED443769

Santa-Cruz-Grau, E. \& Olmedo, A. (2012). Neoliberalismo y creación de 'sentido común': crisis educativa y medios de comunicación en Chile. Profesorado, Revista de Currículum y Formación de Profesorado, 16 (3), 145168. Disponible en: https://recyt.fecyt.es/index.php/profesorado/article/ view/42704/24599, https://www.ugr.es/ recfpro/rev163ART8.pdf

Subirats, J. \& Nogales, I. (1989). Maestros, Escuelas, Crisis educativa. Condiciones del trabajo docente en Bolivia. Santiago de Chile: Organización de las Naciones Unidas para la Educación, la Ciencia y la Cultura, Oficina Regional de Educación para América Latina y el Caribe, UNESCO/OREALC. Disponible en: http://unesdoc.unesco.org/images/0008/000853/085394so.pdf

Wangwe, S. M. \& Rweyemamu, D. C. (2001). Human Resource and Institutional Development in Africa: an Overview. Paper Presented at the First Pan African Capacity Building Forum, Bamako, Mali, 22-24 October. Disponible en: http://tzonline.org/pdf/institutionaldevelopment.pdf 